\title{
El video tutorial como herramienta de educación no formal en estudiantes de Bogotá, Colombia
}

\author{
The video tutorial as a non-formal education tool for students in \\ Bogotá, Colombia
}

Orlando González profesorlando@hotmail.com

http://orcid.org/0000-0002-1330-6020

Universidad de Málaga (España)

\section{Resumen}

Esta investigación buscó conocer cuál es el uso del video tutorial como alternativa para la educación no formal, en estudiantes de los colegios La Amistad y Distrital Kennedy de Bogotá, Colombia (2016-2017). Se diseñó una metodología mixta, que empleó setenta encuestas físicas y ochenta digitales, para una muestra de 150 de un total de 300 educandos. Además de un grupo focal, realizado en dos jornadas de una hora con diez estudiantes de décimo y undécimo grado, en ambos colegios. Los resultados indicaron que la relación de los jóvenes con las nuevas tecnologías favorece el aprendizaje. La mayoría de los estudiantes usan YouTube constantemente para consultar sus actividades académicas y otros contenidos. Esta plataforma contiene una gran cantidad de videos tutoriales de fácil acceso, donde predominó el interés por la investigación, los idiomas y la tecnología, además saber sobre profesiones y oficios. De este modo, se evidenció que la web es una fuente de educación no formal y el video tutorial una excelente opción por sus características didácticas y su diversidad de temáticas. 
Palabras clave: aprendizaje; audiovisual; comunicación; educación no formal; tecnología.

\section{Abstract}

This research sought to know what is the use of video tutorial, as an alternative for non-formal education, in students of La Amistad and Kennedy District schools in Bogotá, Colombia (20162017). A mixed methodology was designed, which used 70 physical and 80 digital surveys, for a sample of 150 out of a total of 300 students. In addition to a focus group, held in two days of one hour with 10 students of tenth and eleventh grade, in both schools. The results indicated that the relationship of young people with new technologies favors learning. Most students use YouTube constantly to check their academic activities and other content. This platform contains a large number of easily accessible tutorial videos, where interest in research, languages and technology predominated, as well as knowledge about professions and trades. In this way, it was evident that the web is a source of non-formal education and video tutorial is an excellent option due to its didactic characteristics and its diversity of topics.

Keywords: learning; audiovisual; communication; non-formal education; technology.

En las últimas décadas ha cambiado la manera de estudiar, aprender y abordar el conocimiento en la era digital, lo que ha permitido nuevas formas de consumo y uso de la tecnología involucrada en los proceso de aprendizaje. En consecuencia, nos encontramos en una sociedad donde es posible formarse fuera de la escuela, con otros tiempos y espacios. La juventud tiene a su disposición diferentes plataformas en Internet para acceder al conocimiento. YouTube ha sido una de las más importantes, demostrando que el medio audiovisual puede ser una herramienta eficaz para encontrar cualquier contenido en la red. Son este tipo de herramientas las que han cambiado la forma de aprender en las más recientes generaciones, la tecnología permite la comunicación, la diversión y la educación. Por esto, es posible considerar que en la actualidad educarse es una opción cada vez más accesible.

En este contexto, YouTube se presenta como una importante alternativa para la educación no formal, que también puede aprovecharse para enseñar o reforzar procesos académicos. El video tutorial es el formato audiovisual al que más acuden los jóvenes, debido a que lo encuentran ameno, de fácil acceso, concreto y práctico para adquirir un conocimiento 
determinado. Además, su formato ofrece una nueva forma interactiva para aprender y enseñar en un medio digital, convirtiéndose en el preferido por los jóvenes para acercarse a conocer diferentes temas de interés.

En este estudio se muestran nuevas opciones para aprovechar la tecnología a favor del aprendizaje, fuera de una educación convencional, a partir del uso que los jóvenes le dan al video tutorial en los colegios La Amistad y Distrital de Kennedy, en Bogotá Colombia. Se presenta esta herramienta como un medio para el proceso de aprendizaje independiente, donde se evidencia la preferencia por lo audiovisual al momento de aprender. Además, con este estudio también se indaga en los contenidos que más buscan los estudiantes y los temas que más les interesa para beneficio de su formación. Por ello, es pertinente esta investigación para saber cómo aprenden los estudiantes desde la educación no formal, a través de la tecnología, para el desarrollo de su formación personal y académica.

\section{Educación no formal desde el video tutorial}

El término educación no formal tiene sus orígenes en la Conferencia Internacional sobre la Crisis Mundial de la Educación que fue celebrada en Williamsburg, Virginia (EE. UU.) en 1967. El analista del Instituto Internacional de la Educación de la UNESCO, Philip Coombs, señala que la educación formal es el sistema educativo institucionalizado, cronológicamente graduado y jerárquicamente estructurado que abarca desde la escuela primaria hasta la universidad. Entre tanto, la educación no formal es "toda actividad educativa organizada, sistemática, realizada fuera del marco del sistema oficial, para facilitar determinadas clases de aprendizajes a subgrupos particulares de la población, tanto adultos como niños" (Homs, 2001). Se marca la diferencia, de la institucionalidad y lo que está al alcance de toda la población para su formación.

En la actualidad, el papel del aprendizaje es fundamental por los cambios tecnológicos que ha transformado la manera de obtener la información, permitiendo a la educación un abanico de posibilidades de generar y acceder al conocimiento.

La educación no formal entonces, se define "como actividades educativas organizadas, sistemáticas, realizadas fuera del marco del sistema oficial” (Martín, 2013: 4). Es un proceso de aprendizaje que incluye prácticas donde las estructuras se escapan de los estándares educativos formales, para constituir una formación de capacitación e información asumida por las personas de acuerdo con sus necesidades educativas. Ante las posibilidades de desarrollo 
que ofrece este proceso, el primer paso esenciales la capacidad educativa de identificar las cualificaciones necesarias para las tareas que se tienen que hacer, así como responder a las inquietudes de dónde buscar dichas habilidades, cómo aprenderlas y cómo aplicarlas (Castells, 1999). "Es posible educar fuera del aula donde el educando pueda interactuar con el medio ambiente, explorar, indagar e investigar y con ello todo lo que implica las actividades del hombre y el mundo físico que lo rodea" (Sánchez y Galvis, 2016: 12). Entonces, como se puede apreciar, el ser humano es inquieto, se interesa por buscar espacios de aprendizaje fuera de los establecidos, para incrementar sus experiencias y enriquecer tanto su proceso de formación como su desempeño en la vida social de acuerdo a sus intereses y particularidades. Con respecto a América Latina, la educación no formal ha tenido una fuerte repercusión en la sociedad "como una vía para producir una ampliación de las posibilidades educativas, y, en tanto práctica, ha supuesto una proliferación de experiencias tendientes a una mayor democratización de la educación" (Nassif, Tedesco y Rama, 1984: 69). Entendemos que este tipo de educación cumple la función de otorgar prácticas en momentos concretos que generen cualquier conocimiento, así como de inclusión, la educación no formal es la que generalmente se da para proveer aprendizaje a subgrupos particulares de la población" (Rivero, 1979: 73). Entre esos grupos los más interpelados son aquellos que requieren ampliar el conocimiento de acuerdo a su labor, estos pueden ser docentes, estudiantes o personas inquietas en seguir aprendiendo, lo importante es contar con una herramienta o sistema como Internet al cual acceder constantemente.

Por su parte, Marenales dice que la educación:

Deberá responder a las nuevas exigencias de la sociedad. Realizar un nuevo tipo de formación centrada en la optimización de recursos y que no se puede pensar en alcanzarla sólo a través de los sistemas actuales. Es imprescindible pensar en medios flexibles, rápidos, baratos e integrados en los circuitos existentes de producción (1996: 8).

Esas exigencias involucran las tendencias de las nuevas tecnologías y abren el debate frente al uso de las mismas, que sí se usan con un propósito lúdico llevan el componente educativo más allá de la escuela, pues la educación, comprende procesos de aprendizaje no formal e informal (Pérez-Tornero y Tejedor, 2014). También se debe entender que dichas tecnologías cumplen funciones educadoras a través de diferentes formatos, medios y lógicas, que pudiendo ser difusas y no intencionadas, resultan ser igualmente educativas. Se trata de involucrar las TIC al aprendizaje de cualquier individuo, que le permite su formación a lo largo de su vida. 
En este sentido, la educación no formal integra a la población a un tipo de educación de mayor accesibilidad y permanencia, que se puede entender como colaborativa para aquellos que están en el marco de una educación formal. Es decir, como un apoyo al proceso de conocimiento que se viene desarrollando en el aula, y como una posible alternativa para la gestión del conocimiento desde un aprendizaje independiente.

Sin embargo, la educación autónoma se presenta por diferentes motivos, ya sea para tener una cultura general o como preparación para integrarse al mundo laboral. Una de sus fortalezas se centra mayoritariamente en "contenidos que abarcan áreas muy específicas del conocimiento y se proponen por lo general, objetivos de carácter instrumental y en corto plazo" (Marenales, 1996: 6). Lo que se refleja es el propósito del uso de la educación no formal, las personas son puntuales a la hora de buscar información con el objetivo de aprender, parte de los intereses personales que pueden ir, desde un curso de belleza hasta un tutorial de técnico automotriz.

Se percibe que la educación no formal es importante, porque ha demostrado que no se requiere de mucha gestión, solo las ganas de querer continuar aprendiendo. La educación no formal permite adquirir y ampliar conocimientos que la educación formal no llega a complementar (Reyes, 2000). Por tanto, la vocación de acceder al conocimiento puede ser permanente, implica una dedicación disciplinaria de actividades de búsqueda de la información pertinente, la cual conllevan al uso de los medios tecnológicos constantemente.

Con las consideraciones anteriores y con las nuevas tecnologías de la información y comunicación, se evidencia más esa disposición para aprender con la red. Lo cierto es que Internet ha facilitado no solo la manera de comunicarse, también la forma de acceder a la información en una autopista interminable de datos, a los que se puede acceder constantemente y donde "muchas personas en todo el mundo aprenden una serie de habilidades cuando utilizan Internet" (Costa, Cuzzocrea y Nuzzachi, 2014: 164). A través de la web se presentan muchas posibilidades, pero parece que el fin educativo es uno de los mayores propósitos de las personas, de igual manera, se usa en las relaciones sociales la comunicación y búsqueda de información entre otras miles de opciones.

Se dice que con la llegada de las tecnologías se abren nuevas posibilidades en todos los campos, especialmente en la educación. Estamos en el momento de transformación social de la información y es "la oportunidad histórica de poder construir un imprescindible trampolín digital que permita dar un salto cuantitativo y cualitativo en el desarrollo económico, social, educativo y cultural" (García-Matilla, 2010: 11). Lo que causaría gran impacto en la sociedad y promete ser una misión visionaria y ambiciosa. En efecto, la educación con las nuevas tecnologías promete ser una oportunidad innovadora con contenidos sintetizados al alcance de 
todos. "Lo fundamental es que las personas nos adaptemos a las nuevas circunstancias mediante el aprendizaje" (Jaraba, 2015: 18). Significa que, aunque se deje la escuela o se haga mayor, la sociedad sigue avanzando y exigiendo una gestión del conocimiento, de un modo $u$ otro, la sociedad se adapta a situaciones nuevas en contextos reales o virtuales, lo importante es el ímpetu de seguir creciendo intelectualmente.

Ahora bien, la investigación muestra que la red sirve como un instrumento de acceso al conocimiento para un fin educativo. A pesar de que en América Latina la brecha digital aún está presente en la problemática social, los cibercafés siguen siendo una gran opción para las personas que no tienen cómo adquirir Internet en sus hogares y buscan la manera para poder acceder al conocimiento, también se destaca el esfuerzo del Estado por expandir el acceso a las TIC y alfabetización digital. El objetivo fundamental es que la tecnología esté al alcance de todo el mundo, "la clave del uso de Internet para potenciar el desarrollo, es la capacidad de las personas de encontrar la información adecuada, analizarla y enfocarla a cualquier tarea que quieran o necesitan" (Castells, 2002: 100).El ciberespacio es más abierto y activo, que genera participación de cualquier persona para beneficio de sus intereses, ya sea producir o recibir mensajes, sin importar cualquier situación social que se presente.

Esto conlleva a entender, que la llegada de la Internet ha cambiado la manera de buscar la información, y con el surgimiento de las redes sociales el cambio en la forma de comunicarse, dando un giro de 360 grados en las relaciones interpersonales. Desde su aparición en 2005 la red social YouTube es aprovechada por los seguidores de la producción audiovisual. YouTube es una plataforma en la cual los usuarios pueden subir y compartir videos. Aloja una gran variedad de clips, películas, programas de televisión y vídeos musicales, así como contenidos de videoblogs y tutoriales. Todo esto soporta la efectividad comunicativa de YouTube, que contiene una gran cantidad de videos que promueven el conocimiento y la interacción.

Aunque YouTube no es el único portal de video online, es el más visitado en la actualidad. Más allá de haber sido el primero de su clase en aparecer, se ha convertido en un referente de la cultura digital de forma casi inmediata. Luego de que fuese adquirido por Google incrementó su dimensión comercial, sus posibilidades tecnológicas y extendió su plataforma. Lo que lo llevó a ser el tercer sitio web más visitado en todo el mundo, detrás del buscador Google y de la red social Facebook (Pérez Rufi, 2013: 45). En este caso, YouTube se ha convertido en el favorito de los realizadores audiovisuales, y por ende, de quienes lo usan para enseñar a través del video tutorial.

Esta plataforma digital es aprovechada por los jóvenes, que continúan respondiendo desde los nuevos escenarios a los mismos retos y eventualidades que sortean cotidianamente. Además 
de nuevas situaciones como el maltrato anónimo o ciberbullying. Cabe resaltar que esta generación, "ni siquiera ha conocido otra generación que la de Internet" (Ferrer, 2010: 42). No le gusta la formalidad, tiende a ser amigable y flexible. También, estos jóvenes están más preparados y cualificados que sus generaciones anteriores, pues cuentan con el conocimiento generado y promovido por la web. Estas características facilitan la interacción y promueven una sensación de comunidad, moldeando nuevas formas de asociación y comportamiento entre los individuos. Todo lo anterior se evidencia en múltiples dinámicas juveniles que pueden ser observadas en ambientes laborales y educativos.

Ahora bien, la plataforma presenta una gran variedad de videos, por ello, es importante reconocer el trabajo de los realizadores que publican en YouTube, que son diversos y "los clasifican en dos grupos: los que son creados por las industrias culturales y aquellos desarrollados por los youtubers -entendidos como usuarios generadores de contenidos gracias a las posibilidades que brinda la Web 2.0" (Murolo y Lacorte, 2015: 20). El propósito de cada productor audiovisual depende de sus mensajes y a quién va dirigido. También se diferencian en la calidad de la producción, unos son más rudimentarios y otros mantienen la calidad que la industria exige.

De esta manera, en YouTube podemos encontrar una diversa gama de videos. Los cuales mantienen la forma clásica de narrar el audiovisual, pero con la convergencia digital han surgido nuevos formatos, estos son: el remix, el lip-dub, el videoblog testimonial o vlog, los webisodes, los sketches, los tutoriales, los gameplays y los registros documentales (Rufi y Pérez, 2013). Todos tienen gran aceptación en los jóvenes, pero es el video tutorial el formato más buscado, puesto que su versatilidad permite abordar una gran cantidad de temáticas a través de diferentes propuestas audiovisuales, facilitando además su búsqueda.

Los videos tutoriales caracterizan la figura del youtuber, quien graba sus propias piezas sobre toda clase de temas, por ejemplo: pruebas de productos, cómo solucionar un problema informático, cómo utilizar programas de software, indicaciones sobre maquillaje, recetas de cocina o reparación de cualquier tipo de dispositivo. Es por ello que en el campo educativo el video tutorial puede ser usado para el aprendizaje de idiomas, el uso de métodos investigativos, la realización de reseñas de libros o películas y hasta la ejecución de procesos industriales.

El video tutorial es una herramienta ideal para adquirir conocimiento y por ello son los más buscados en YouTube "son tan eficaces y válidos como los cursos presenciales. Por ejemplo, los videos que utilizan capturas de pantalla con audio para la enseñanza del uso de software" (Castillo y Carrillo, 2012: 69). Lo anterior se da porque para aprender, la imagen y el sonido se 
vuelven atractivos y otorgan mayor atención para captar los mensajes (Mayer, 2001). El video tiene esa característica especial que ilustra lo que se está contando, de ver como se hace, poderlo grabar y después reproducir al antojo del usuario.

Por consiguiente, se puede decir que el video tutorial favorece al aprendizaje, siempre que conlleve una serie de elementos:

\begin{abstract}
Debe favorecer la realimentación, comprobación, aplicación, demostración, resolución de ejercicios, problemas de la vida diaria y proyectos de una manera interactiva brindando un juego de iniciativas a través de organizadores gráficos y animaciones hacia la búsqueda de fundamentación científica y su ejecución, conseguir además un aprendizaje significativo que implica un cambio en los esquemas de conocimientos que se poseen previamente, estableciendo nuevas relaciones entre dichos elementos, mejorando de esta manera el proceso de enseñanza-aprendizaje (Fernández, Díaz, Del Carmen y Recio, 2013: 1994).
\end{abstract}

Lo anterior examina con juicio el proceso que debe tener un video tutorial, para que logre un impacto favorable debe considerarse el buen uso del lenguaje audiovisual, con todos sus elementos visuales que apoyen la explicación de lo que se está enseñando. Además de cumplir con la rigurosidad de la investigación para tratar el tema expuesto frente a cámara.

El uso del video tutorial no es exclusivo de la academia, como se ha expuesto antes; todos pueden tener acceso a este tipo de audiovisual para su formación. "Lo anterior nos lleva a examinar la utilidad de los videotutoriales como herramientas que superan la limitación de la presencialidad obligatoria" (Castillo y Carrillo, 2012: 69). La necesidad de seguir aprendiendo dentro y fuera del aula es lo que lleva a cualquier persona a estar interesado por la educación. La tecnología ha permitido superar la presencia en la escuela facilitando la continuidad de adquirir el conocimiento de manera autónoma.

En efecto, el video tutorial tiene un devenir bastante promisorio, tanto así que estos favorecen un aprendizaje significativo, al establecer nuevas relaciones que mejoran el proceso de enseñanza-aprendizaje (Márquez, 1995). Se evidencia que estamos frente a una poderosa herramienta audiovisual. El video tutorial aporta esa posibilidad de reforzar el proceso de enseñanza a cualquier estudiante o persona que viva inmersa en las pantallas digitales buscando, para diferentes propósitos, ampliar su conocimiento.

Tomando en cuenta el curso de Estadística II en el periodo de febrero-julio de 2012, las investigaciones de Mario Saucedo Fernández, Juan José Díaz Perera, Santa del Carmen Herrera Sánchez, Carlos Enrique Recio Urdaneta de la Universidad Autónoma del Carmen en México, quienes plantearon potenciar el rendimiento académico en los alumnos, así como 
propiciar la construcción de su propio aprendizaje mediante los videos tutoriales. Demostraron que es más cómodo, práctico e interesante tener material en video que ir a la biblioteca, igualmente, manejan mejor los conceptos aquellos que se apoyaron en videos.

Otro estudio lo hace María Isabel Ramírez-Ochoa, con las posibilidades del uso educativo de YouTube, entre julio y diciembre de 2016 en la Universidad Autónoma Indígena de México. El trabajo analiza los atributos educativos alcanzados en la plataforma de YouTube, cuyos resultados mostraron que al buscar la palabra Educación nos brinda más de 3.450 .000 resultados; mientras Education más 11.000 .000 , pero la mayoría reproducen los videos es para ver música $(31 \%)$, entretenimiento (15\%) y sociales (11\%). La educación apenas alcanza el $4.1 \%$ de los videos. Evidencia de que YouTube es un espacio propicio para la investigación del impacto del audiovisual en la enseñanza.

En Colombia, de acuerdo al trabajo de Juan Carlos Gómez Gómez, en 2014 sobre el "videos educativos de YouTube para la enseñanza de las ciencias naturales en educación básica colombiana", resalta la importancia del uso de los videos en la educación, pues resultan favorables en el proceso de aprendizaje y se plantea la siguiente pregunta: ¿son los videos educativos medios de mayor efectividad en el aprendizaje que las prácticas educativas expositivas de corte magistral? Según el portal iab.colombia (2017), ComScore, líder en medición del mundo digital, reveló los siguientes datos sobre el mercado digital en el país:

-El 26\% de la población online en Colombia tiene entre 15 y 24 años.

-Bogotá concentra una cuarta parte de los usuarios de todo el país, mientras que Antioquia, Atlántica y Oriental, en partes casi iguales, comparten el segundo lugar.

-Las principales categorías en el consumo de contenido online son: Servicios, Navegación/Investigación, Entretenimiento, Redes Sociales y Portales.

$-40 \%$ de los colombianos utiliza más de un dispositivo para conectarse a Internet. La población digital del país supera los 33 millones de usuarios entre Desktop, Smartphones y Tablets.

Bajo estos supuestos relevantes concebimos el propósito de esta investigación, que nos permite conocer la motivación de los estudiantes por el video tutorial como actividad de aprendizaje para complementar su formación académica. En este sentido, podemos llegar a conocer otras formas de aprender, en otros ambientes nuevos donde se enriquece la educación, a través del audiovisual, como herramienta didáctica que impacta y seduce a los estudiantes. Específicamente, esta investigación tiene dos objetivos: el primero es conocer cuál 
es el uso del video tutorial, como alternativa de educación no formal, y el segundo, saber cuáles son los contenidos que más les interesa ver a los estudiantes bogotanos.

\section{Metodología}

Se empleó una metodología que aborda una perspectiva mixta (cualitativa y cuantitativa), centrada en una investigación de tipo descriptivo. Esto nos permite obtener una visión holística sobre el estudio del fenómeno. Se contó con la colaboración del colegio Distrital Kennedy conformado por más de 140 estudiantes en los grados de décimo y undécimo, y el colegio La Amistad, que cuenta con más 160 alumnos de los mismos grados. Ambos colegios pertenecen al mismo sector de Bogotá, del Barrio Kennedy. El número de jóvenes que conformó la muestra fue de 150 alumnos de décimo y undécimo grado de secundaria, quienes tenían entre 14 y 20 años de edad. El 47,6\% de los participantes eran mujeres y el 52,4\% hombres. Se aplicaron las técnicas de encuesta y grupo focal de la siguiente manera: el primer instrumento, que era la encuesta, se aplicó personalmente en ambos colegios, logrando una participación de setenta estudiantes que la diligenciaron en papel y ochenta de forma digital a través del correo electrónico, para un total de 150 encuestas. Posteriormente, se realizó un grupo focal, que fue grabado mediante un celular móvil. Primero se realizó en el colegio La Amistad, con una duración de una hora y con la participación de diez estudiantes de undécimo grado. Posteriormente, en el colegio Distrital Kennedy se llevó a cabo durante media hora, con diez jóvenes de décimo grado.

\section{Resultados}

Los resultados alcanzados demuestran el gran interés que tienen los estudiantes colombianos por el uso de las tecnologías digitales que fortalecen el aprendizaje autónomo. En esta investigación se puede apreciar que la mayoría de jóvenes (85\%) se interesa por el video tutorial como medio de enseñanza no formal. Esta herramienta tecnológica es utilizada fuera de la academia para tener un conocimiento principalmente orientado a la formación personal. Según indican los estudiantes, porque es una herramienta audiovisual que los motiva a descubrir nuevos conocimientos y ampliar los ya adquiridos. 


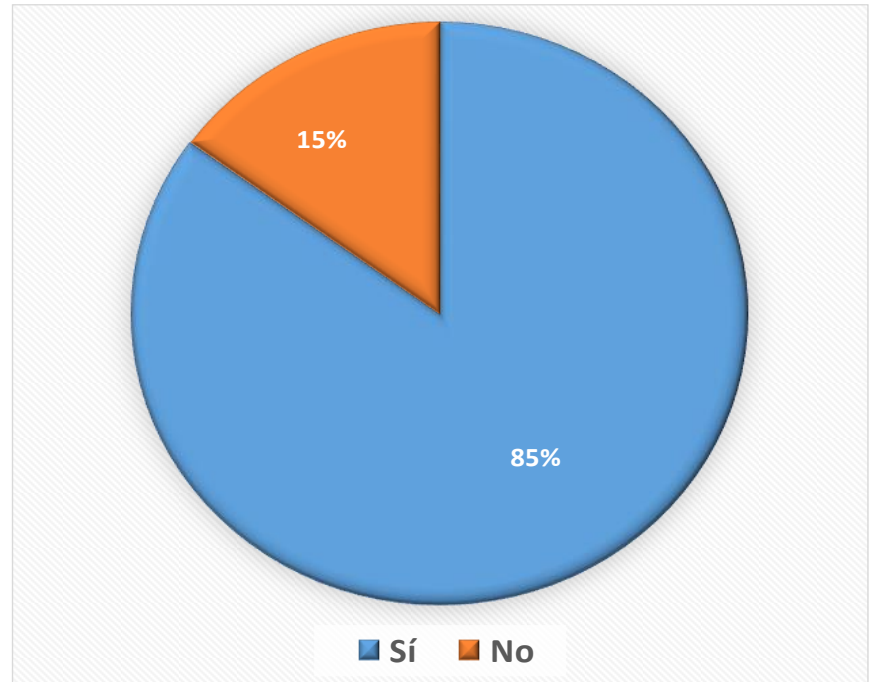

Gráfico 1: ¿Utiliza el video tutorial como proceso de aprendizaje no formal?. Fuente: elaboración propia

Los resultados obtenidos en la encuesta son contundentes. Demuestran que un gran porcentaje del uso del video tutorial se da como herramienta de aprendizaje no formal. En cuanto a la preferencia de las redes, los estudiantes utilizan mayormente la plataforma de YouTube como la red audiovisual más importante para visualizar los videos. Especialmente porque la información visual y sonora les resulta más atractiva para aprender.

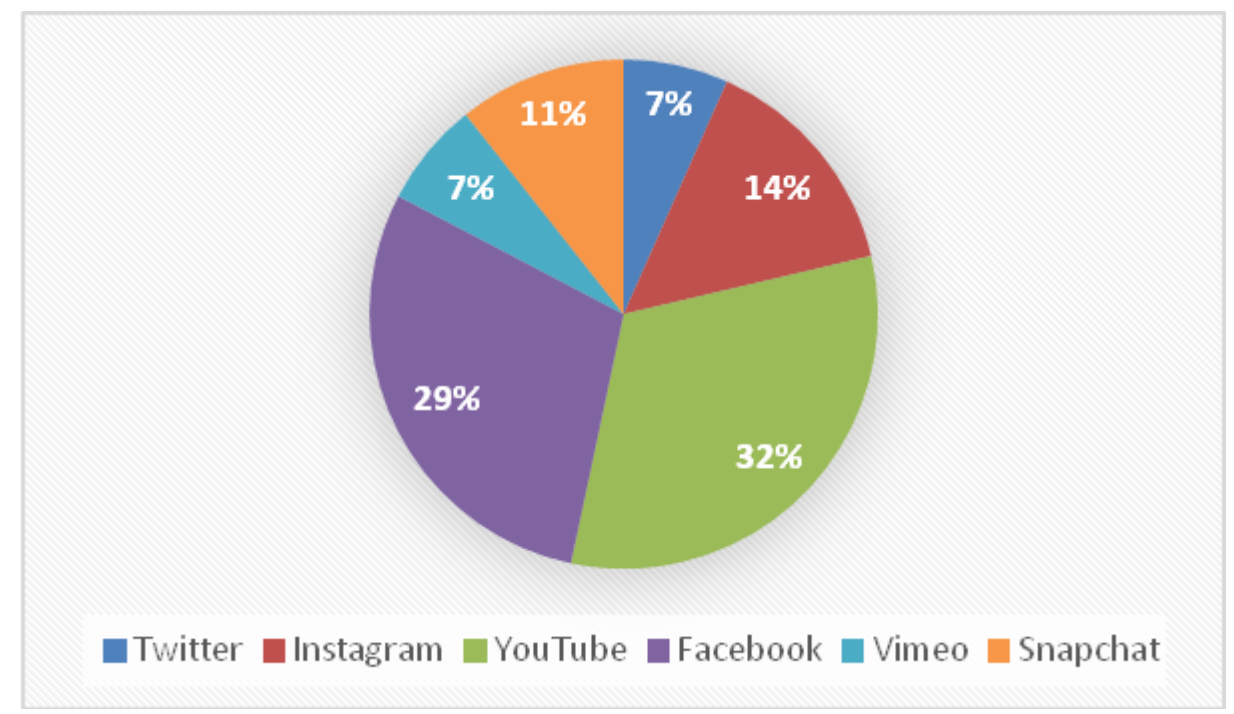

Gráfico 2: Redes sociales de mayor preferencia. Fuente: elaboración propia. 
Como se presenta en el Gráfico 2, el 32\% de los muchachos prefieren a YouTube sobre las otras redes sociales. Le sigue Facebook con el $29 \%$, donde también se comparten videos y con el 14\% Instagram, que también integró la función audiovisual. En el 11\% ubican Snapchat, una red dedicada enteramente a la creación de videos cortos con efectos y en el último lugar con el 7\% cada una, identifican Twitter y Vimeo.

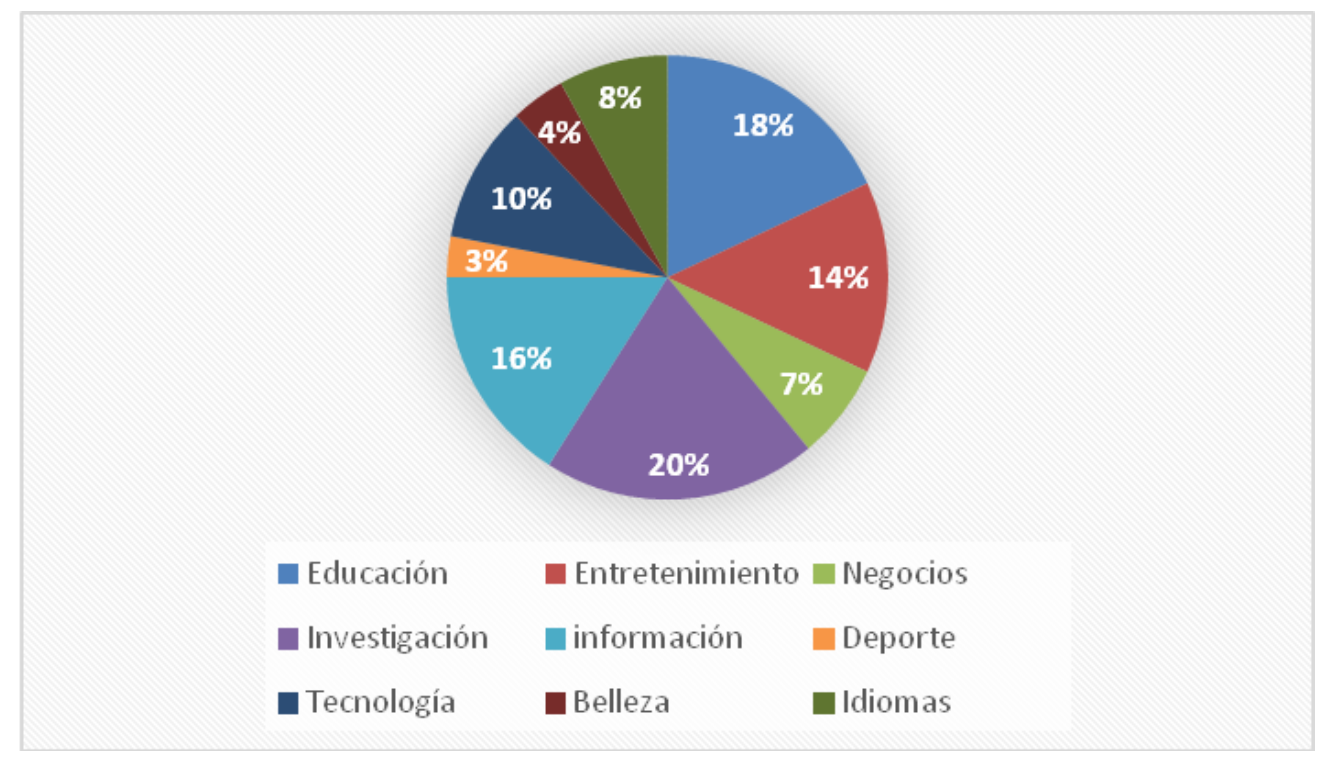

Gráfico 3: ¿Cuáles son los contenidos que más buscan en YouTube? Fuente: elaboración propia.

Con respecto a los contenidos más buscados en YouTube, los estudiantes siguen prefiriendo la investigación con un $20 \%$, la educación con un $18 \%$ y la información general con un $16 \%$, por encima de otras como entretenimiento $14 \%$; tecnología $10 \%$; idiomas $8 \%$; negocios $7 \%$; deporte $3 \%$ y belleza $4 \%$. Estos contenidos conciernen a los tratados en la escuela o temas particulares que el estudiante busca en la red social YouTube. 


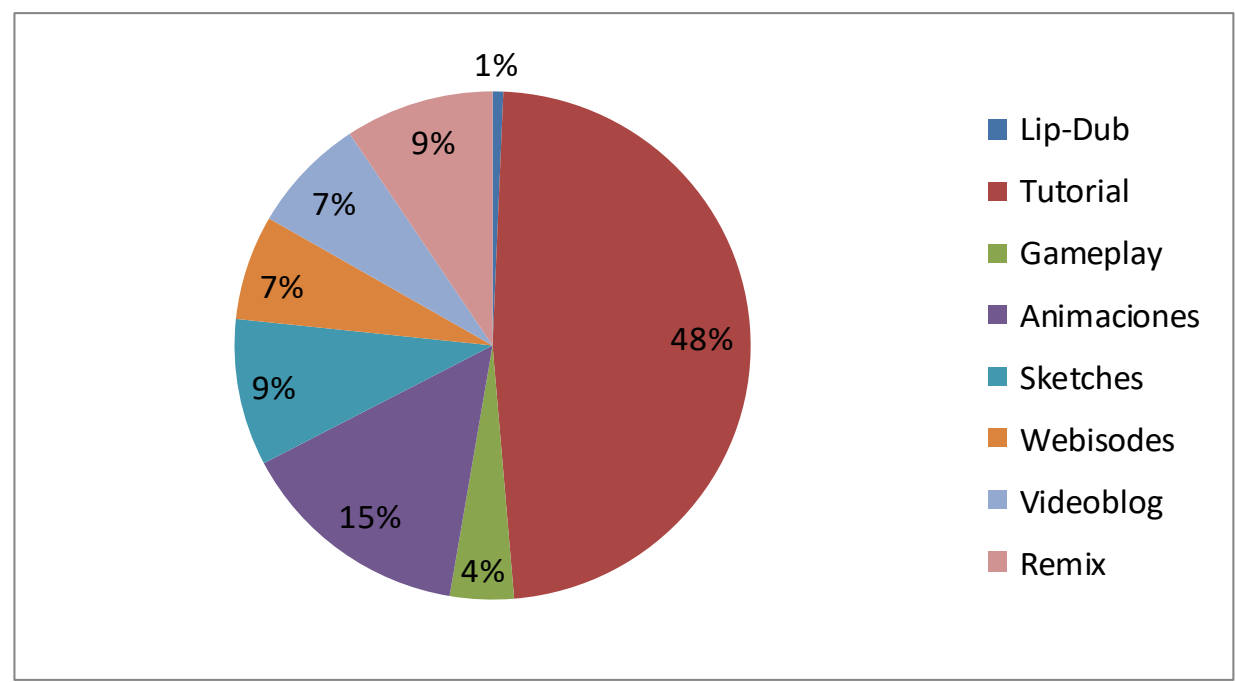

Gráfico 4: ¿Formato audiovisual digital más requerido? Fuente: elaboración propia.

Dentro de los formatos más utilizados por los estudiantes, queda claro que el tutorial es al que más acuden según indican los resultados. Como se presenta en el Gráfico 4, el tutorial es utilizado por el $48 \%$ de los estudiantes, seguido por las animaciones, con un $15 \%$. Los restantes cuentan con porcentajes bastantes inferiores, Sketches 9\%, Remix 9\%, los Webisodes 7\%, Videoblog 7\%, Gameplay 4\% y Lip-Dub 1\%. El tutorial es más buscado porque les permite conocer sobre diversos temas de una manera más lúdica. Los estudiantes ven que los contenidos y procesos son sencillos de seguir, gracias a que el video tutorial permite repetir, pausar y guardar las veces que sean necesarias, lo que conlleva a un proceso de aprendizaje más claro y directo. 


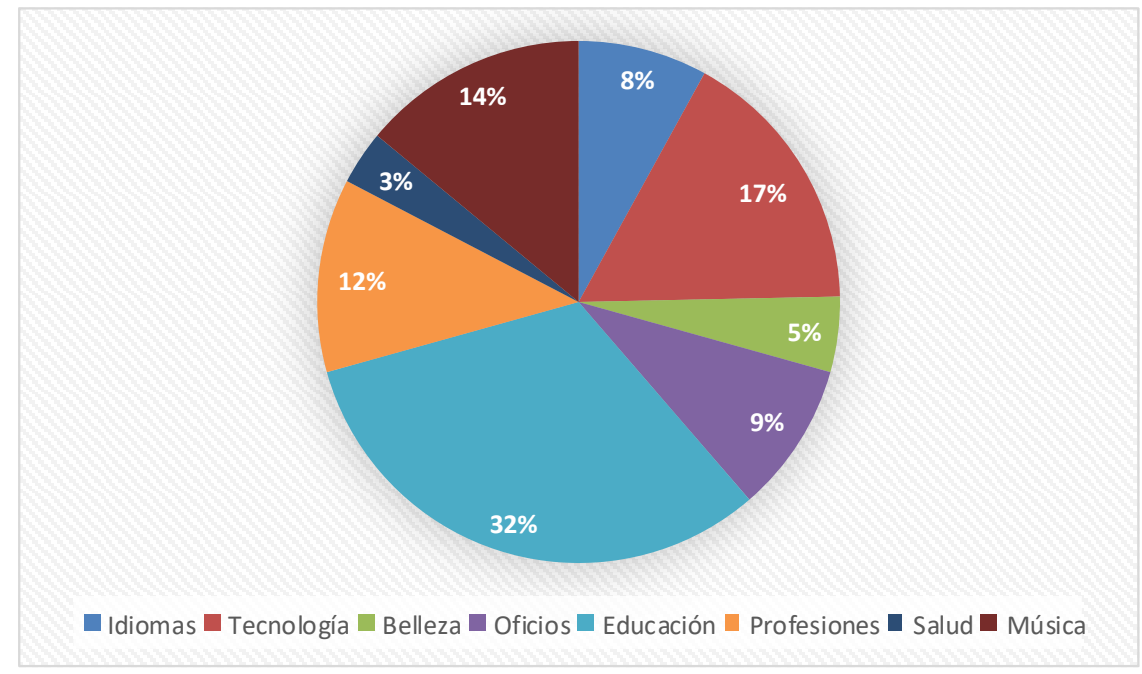

Gráfico 5: ¿Qué contenidos son los que más buscan en los videos tutoriales? Fuente: elaboración propia

Por otra parte, en cuanto a los contenidos que más buscan los estudiantes en los videos tutoriales, las temáticas educativas son las más relevantes con un $32 \%$, seguidas en orden descendente por las temáticas de tecnología con 17\%, música con 14\%, profesiones $12 \%$, oficios $9 \%$, idiomas $8 \%$, belleza $5 \%$ y salud $3 \%$. Estos resultados nos indican que hay una tendencia de los estudiantes, fuera de clases, a seguir interesados en los contenidos sobre educación y tecnología. Además, cabe resaltar que el interés que se revela por los temas de profesiones u oficios seguramente está marcado por los intereses vocacionales que tienen los estudiantes para su futuro como profesionales.

El uso de YouTube y del video tutorial es primordial para los estudiantes, que no dejan de visitar esta red social para ver videos con contenidos informativos que les sirve como herramienta tecnológica para el apoyo de la educación formal, desde una visión educativa no formal. De esta manera, el video tutorial ha logrado un gusto muy difundido por los jóvenes que comparten e interactúan en un medio digital fundamental para acceder al conocimiento.

Ahora bien, mediante el grupo focal los estudiantes evidenciaron la importancia del uso de YouTube y el video tutorial, reconociéndolos como medios de mayor relevancia que la televisión. Estos jóvenes dejan de ser consumidores pasivos y adquieren libertad para conocer todo tipo de contenidos actuales, en cualquier momento y lugar. A partir de una serie de preguntas se llevaron a cabo dos sesiones que nos demuestran el interés a la hora de usar el video tutorial como herramienta de educación no formal por parte de los estudiantes bogotanos. Para ello, seguimos las sugerencias de Hamui-Sutton y Varela-Ruiz (2013) de elaborar un primer árbol categorial basado en la guía de la entrevista. Desde códigos se forman 
unidades, categorías y patrones con el fin de explicar contextos, situaciones, hechos y fenómenos. De esta forma realizamos un cuadro de categorías que se presentan en la Tabla 1. Estas surgieron del grupo focal y nos dieron una visión de las dimensiones de uso para identificar prácticas, aspectos y fines del video tutorial

\begin{tabular}{|l|l|}
\hline \multicolumn{1}{|c|}{ Categorías } & \multicolumn{1}{c|}{ Dimensiones } \\
\hline Prácticas de uso & $\begin{array}{l}\text { Almacenar-compartir-interacción-tiempo- } \\
\text { motivador-curiosidad-efectividad-cualquier lugar y } \\
\text { momento. }\end{array}$ \\
\hline Aspectos del uso & $\begin{array}{l}\text { Complemento de educación formal-dinamizador- } \\
\text { recurso didáctico-refuerza el aprendizaje- } \\
\text { rendimiento académico-contenidos-tarea de aula- } \\
\text { alternativa a la educación formal y ver haciendo. }\end{array}$ \\
\hline Fin del uso & $\begin{array}{l}\text { Instrumento para aprender- medio de } \\
\text { información-instrumento para investigar- amplia el } \\
\text { conocimiento-obtener habilidades y destrezas- } \\
\text { adquirir competencias-dominar un tema y auto } \\
\text { aprendizaje. }\end{array}$ \\
\hline
\end{tabular}

Tabla 1: Cuadro de categorías del uso del video tutorial. Fuente: elaboración propia 2017.

En el análisis de las categorías, YouTube despierta pasiones en los gustos de los muchachos, pues constantemente están relacionados con los medios audiovisuales. El video tutorial para los estudiantes es de gran relevancia porque actúa como fuerza de motivación en lo referente a descubrir y compartir el conocimiento. Por las consideraciones anteriores, encontramos que lo que más se destaca del uso del video tutorial, como recurso de educación no formal entre los jóvenes estudiantes de los colegios La Amistad y Distrital Kennedy de Bogotá, es la motivación de esta generación digital, para buscar la información, ampliar el conocimiento y continuar la 
educación formal, además de ser un recurso de investigación de interés personal determinado a la formación humana y social (Tabla 1).

Las discusiones de los estudiantes se centran más en elegir el tema del video, que en elegir la técnica con la que se elabora, aunque le dan gran importancia a la alta definición. Lo que más los lleva a usar este tipo de formato de video, como lo es el tutorial, es aprender procesos, conceptos y otros temas que les interesan, pero que no se tocan en clases o son muy difíciles de comprender en espacios diferentes. Otros factores que favorecen su uso para los estudiantes es el costo, el video se convierte en un aliado para aprender sin ningún valor monetario, puesto que permanece al alcance de todos por medio de cualquier dispositivo electrónico.

En cuanto a los contenidos, para las mujeres los videos tutoriales de belleza permiten mejorar su propia apariencia; así como aprender acerca del oficio de la estética. Para los hombres los temas más mencionados son la tecnología, la música y los deportes. Sin embargo, la valoración más importante que le otorgan los estudiantes al video tutorial está en su uso como recurso didáctico de aprendizaje. Lo catalogan como un factor primordial en la enseñanza, porque el video tutorial facilita el rendimiento escolar gracias a que suelen presentar los contenidos de forma corta, sencilla y directa. También sirve como herramienta para lograr los propósitos de auto aprendizaje, adquirir competencias y enfrentar a la sociedad de manera competitiva. Pero todo se debe a las características atractivas del audiovisual, la fascinación del audio y de la imagen, que ofrecen una experiencia más contundente que cualquier otra herramienta pedagógica.

Con respecto a la práctica de uso, es significativo para los estudiantes, este formato brinda el poder de almacenarlo y compartirlo para reproducirlo en cualquier momento y verlo detenidamente para un proceso de aprendizaje más eficaz. De esta manera, la enseñanza que permite el video tutorial toma fuerza en todos los ámbitos, pues no solo refuerza la escolaridad, también, funciona como instrumento alternativo en la educación no formal. En la plataforma de YouTube se adquiere información, conocimientos y competencias que dejan en los muchachos la inquietud de saber muchas cosas $o$ aprender un arte $u$ oficio para su desarrollo personal y profesional, logrando también siendo atractivo y repetitivo.

El video tutorial tiene especial acogida entre los estudiantes; el estar conectados a los dispositivos tecnológicos produce nuevos hábitos frente a los medios. Desde la enseñanza, estas herramientas se multiplican pero ¿realmente son eficaces en el proceso de aprendizaje? ¿Qué otros medios tecnológicas están aportando a la educación? Se presentan muchas 
inquietudes respecto al uso de las nuevas tecnologías en la educación y se plantea el debate frente a la gestión del conocimiento.

\section{Conclusiones}

Los resultados obtenidos en esta investigación nos muestran la realidad de los jóvenes estudiantes enmarcados en múltiples entornos digitales. Las pantallas se multiplican, hay mayor facilidad de acceder a la información en comparación con décadas anteriores. Para aprender ya no se necesita acudir a un salón de clase (Costa, Cuzzocrea y Nuzzachi, 2014). Internet nos ha puesto a disposición cualquier cantidad de aplicativos, páginas web y redes sociales para hacerlo. Estas herramientas digitales han sido aprovechadas por los muchachos para continuar su proceso de aprendizaje.

Con base en los resultados cuantitativos, el video tutorial tiene un $85 \%$ de interés por parte de los estudiantes de los colegios bogotanos La Amistad y Distrital de Kennedy, que lo usan como recurso de educación no formal. Los jóvenes utilizan esta herramienta por las posibilidades que permite el formato audiovisual de aprender con mayor facilidad y diversidad de contenidos.

Se percibe que el video tutorial permite el acceso a todo tipo de conocimiento, siendo de preferencia, en primer lugar, aquellos temas que tocan la educación con el 32\% y la tecnología con el $17 \%$, siguiendo en importancia otras áreas específicas donde predominan temas como idiomas, música, belleza y deportes, que guardan un valor de entretenimiento e información a la hora de elegir otros contenidos alternos a lo educativo.

En cuanto a la investigación cualitativa, según el grupo focal, el uso de los videos tutoriales los motiva a conocer y ampliar temáticas relevantes de acuerdo a sus necesidades escolares. Los jóvenes acuden a este formato audiovisual por la facilidad de acceso, la inmediatez y que los pueden compartir por cualquier red social.

También cabe resaltar la utilización de esta herramienta audiovisual, por parte de los estudiantes colombianos, para conocer acerca de diferentes profesiones y oficios. Lo anterior, con el propósito de averiguar todo lo posible acerca de las carreras universitarias, lo que les ayudará en la toma de decisiones sobre su futuro profesional o laboral. El conocimiento en general se distribuye por internet, pero los jóvenes son hábiles para identificar lo que más les llama la atención, eligen algunos contenidos por encima de otros de acuerdo con sus características y prioridades personales (Devaney, 2009). Por ejemplo, según su edad pueden estar buscando videos sobre el futuro académico y laboral. 
En otros resultados que se obtuvieron en esta investigación, se destacan las características que deben tener los videos para ser atractivos para los jóvenes. De acuerdo con lo expresado por los estudiantes, el video debe ser de corta duración, bien ordenado, contar con ayudas gráficas o efectos visuales y enseñar paso a paso mediante una explicación pausada, pero no demasiado lenta. Además, es importante motivar a quien narra o presenta el video, a través de visitas o likes. Un video tutorial con una producción audiovisual bien realizada en técnica y contenido puede llegar a alcanzar millones de visitantes y convertirlo en viral, lo cual le da gran reconocimiento en la comunidad digital.

Es importante señalar que los estudiantes tienen las herramientas necesarias con inmediatez, sin un tutor o guía para su educación, esto lleva a reconocer la velocidad de la interacción en la información y el conocimiento, con un alto grado de auto aprendizaje, pero la volatilidad puede ser un componente de dispersión. "Los jóvenes cada vez saben más (aunque no necesariamente del "currículum oficial") y aprenden más cosas fuera de los centros educativos" Graells, 2013: 4). Vale destacar la disposición de los estudiantes para discernir entre lo transformador y lo superfluo. Sin embargo, el video tutorial favorece a quienes buscan una alternativa de educación no formal, aprovechando las tecnologías de la información, que cada vez promueven la innovación y el acercamiento al conocimiento, mejorando los procesos de enseñanza y aprendizaje. Se observa que profesores, técnicos, estudiantes y cualquier persona, suben diariamente miles de estos videos para disposición de aquellos que quieren aprender constantemente sin el rigor de la enseñanza tradicional o bien como complemento a la escuela. El auto aprendizaje es un elemento de cambio en la sociedad actual, que influye en el ser humano la capacidad de superarse constantemente, en un mundo revolucionado por las tecnologías digitales.

\section{Bibliografía}

Alexa, R. (2011). Informe Tráfico Anual. Recuperado de https://www.alexa.com/siteinfo

Castells, M. (1999). La era de la información: economía, sociedad y cultura (Vol. 1). Madrid: Alianza editorial.

Castells, M. (2002). Tecnologías de la información y la comunicación y el desarrollo global. Revista de economía mundial, 7, pp. 91-107. doi: 10.2307/20645021.

Castillo, D. J. y Carrillo, G. M. M. (2012). Asimilación de contenidos y aprendizaje mediante el uso de videotutoriales. Enseñanza \& Teaching, 30(2), 63-79. 
Coombs, P. y Ahmed, M. (1973). Perspectivas de UNESCO. III(3), Madrid.

Costa, S.; Cuzzocrea, F. y Nuzzaci, A. (2014). Use of the Internet in Educative Informal Contexts. Implicationfor Formal Education. Comunicar, 43, 163-171. Recuperado de https://doi.org/10.3916/C43-2014-16

Devaney, T. A. (2009). Impact of video tutorials in an online educational statistics course. Journal of Online Learning and Teaching, 5(4), pp. 600-608.

Fernández, M. S.; Díaz, J. J.; Del Carmen, S. y Recio, C. E. (2013). El video tutorial como alternativa didáctica en el área de matemáticas. Uso de los recursos tecnológicos en el proceso de aprendizaje de las matemáticas. Comité Latinoamericano de Matemática Educativa.

Recuperado

de http://funes.uniandes.edu.co/4582/1/SaucedoElvideoALME2013.pdf

Ferrer, A. (2010). Millennials, la generation del siglo XXI. Nueva Revista de Política, Cultura y Arte, 130.

García-Matilla. A. (2010). Publicitar la Educomunicación en la universidad del siglo XXI. En García A. R. eds. Más allá Educomunicación del 2.0 (pp. 318-320). Barcelona: Gedisa.

Gómez, J. C. G. (2014). Videos educativos de youtube para la enseñanza de las ciencias naturales en educación básica colombiana. Escenarios, 1(14), 56-80.

Graells, P. R. M. (2013). Impacto de las TIC en la educación: funciones y limitaciones. 3 c TIC: cuadernos de desarrollo aplicados a las TIC, 2(1), 2.

Homs, M. I. P. (2001). Orígenes y evolución del concepto de educación no formal. Revista española de pedagogía, pp. 525-544.

Jaraba, G. (2015). Youtuber, cómo crear videos de impacto y triunfar con ellos en Internet. Barcelona: Editorial ManonTroppo.

Hamui-Sutton, A. y Varela-Ruiz, M. (2013). La técnica de grupos focales. Investigación en educación médica, 2(5), 55-60.

Interactive Advertising Bureau Colombia (2017). comScore presenta "10 datos sobre el mercado digital en Colombia". Recuperado de http://www.iabcolombia.com/loultimo/comscore-presenta-10-datos-mercado-digital-colombia/

Marenales, E. (1996). Educación formal, no formal e informal. Temas para concurso de maestros. Bogotá: Editorial Aula.

Márquez, P. (1995). Software educativo; guía de uso y metodología de diseño. Barcelona: EMAestudio.

Martín, R. B. (2013). Contextos de aprendizaje. Formales, no formales e informales. IKASTORRATZA e-Revista de Didáctica, 12, 1-14. 
Mayer, R. E. (2001). Multimedia Learning. New York: Cambridge University Press.

Murolo, N. y Lacorte, N. (2015). De los Bloopers a los Youtubers. Diez años de Youtube en la Cultura Digital.

Recuperado

de http://perio.unlp.edu.ar/ojs/index.php/question/article/view/2407/2128

Nassif, R.; Tedesco, J. C. y Rama, G. W. (1984). Sistema Educativo en América Latina. Buenos Aires: Editorial Kapelusz.

Pérez Rufi, J. P. (mayo 2013). La actualidad en YouTube: claves de los vídeos más vistos durante un mes. Global Media Journal México, 9(17), pp. 44-62. Recuperado de https://gmjei-ojs-tamiu.tdl.org/gmjei/index.php/GMJ_El/article/view/43/43

Pérez Rufí, J. P. y Gómez Pérez, F. G. (2013). Nuevos formatos audiovisuales en Internet: cuando el usuario es quien innova. En Mira Pastor, E. y de Salas Nestares, M. I. Prospectivas y tendencias para la comunicación en el siglo XXI (pp. 167-187). Madrid: CEU Ediciones.

Pérez-Tornero, J. M. y Tejedor, S. (2014). Guía de tecnología, comunicación y educación para profesores: preguntas y respuestas. Barcelona: Ed UOC.

Ramírez-Ochoa, M. I. (2016). Posibilidades del uso educativo de youtube. Ra Ximhai, 12(6), julio-diciembre, pp. 537-546. Universidad Autónoma Indígena de México. Recuperado de http://www.redalyc.org/articulo.oa?id=46148194036

Reyes, J. (2000). La escuela sola no hará el milagro: el papel de la educación no formal. Memoria del Foro Nacional de Educación Ambiental. México, Universidad Autónoma de Aguascalientes, pp. 43-54.

Rivero H. J. (1979). Educación no-formal en la reforma peruana. Buenos Aires: CEPAL.

Sánchez, G. L. A y Galvis, M. L. P. (2016). Fuera del aula: ambientes divertidos para un aprendizaje significativo. Recuperado de http://hdl.handle.net/10656/4777

Saucedo, M.; Díaz, J. J.; Herrera, S. y Recio, C. E. (2013). El video tutorial como alternativa didáctica en el área de matemáticas. En Flores, R. (Ed.). Acta Latinoamericana de Matemática Educativa (pp. 1991-1999). México, DF: Comité Latinoamericano de Matemática Educativa. 